\title{
Niveles dietarios de metionina y ácido linoleico para codornices criadas en Cajamarca-Perú
}

\section{Dietary levels of methionine and linoleic acid for quails bred in Cajamarca-Peru}

\author{
Manuel Paredes ${ }^{1 *}\left(\mathbb{D}\right.$, Wuesley Alvarez ${ }^{1}$, Luis Vilela ${ }^{1}$, Luis Vallejos ${ }^{1}\left(\mathbb{D}\right.$, José Mantilla ${ }^{1}$ (D)
}

\section{RESUMEN}

El experimento se llevó a cabo en la granja avícola de la Universidad Nacional de Cajamarca, durante veinticuatro semanas para determinar efectos de los niveles dietarios de metionina y ácido linoleico sobre la producción y la calidad del huevo de codorniz (Coturnix coturnix var. japónica). Se inició la evaluación con 576 aves de 42 días de edad, alojadas en jaulas distribuidas según nueve combinaciones de tratamientos, con cuatro repeticiones de 16 aves por cada tratamiento, considerando tres niveles de metionina $(0,40,0,50$ y $0,60 \% \mathrm{MET})$ y tres niveles de ácido linoleico $(1,63,2,05$ y 2,47\% LIN). El rendimiento productivo y la calidad del huevo se analizaron según diseño completamente randomizado en arreglo factorial de $3 \times 3$. Las medias se compararon mediante prueba de Tukey. Las aves alimentadas con dietas con 0,50 y $0,60 \%$ de MET tuvieron mayor ingesta de alimento y producción de huevos (p $<0,05)$ que aquellas alimentadas con dietas con $0,40 \%$ de MET. Hubo interacción significativa $(\mathrm{p}<0,05)$ entre la MET y los niveles de Lin en el peso del huevo. Se observó una mejor conversión alimenticia y masa de huevo para las aves alimentadas con dietas con $0,60 \%$ de MET en comparación con aquellas alimentadas con dietas con $0,40 \%$ y $0,50 \%$ de MET. La producción y la calidad del huevo no mejoraron ( $\mathrm{p}>0,05)$ con los niveles altos de LIN.

Palabras claves: metionina, ácido linoleico, productividad de la codorniz ponedora, calidad de huevo.

\begin{abstract}
The experiment was carried out in the poultry farm of the National University of Cajamarca, during twenty-four weeks to determine effects of the dietary levels of methionine and linoleic acid on the production and egg quality of the quail (Coturnix coturnix var japónica). The evaluation was started with 576 birds of 42 days of age, housed in cages distributed in nine combinations of treatments, with four repetitions of 16 birds per treatment, considering three levels of methionine $(0.40,0.50$ and $0,60 \% \mathrm{MET})$ and three levels of linoleic acid (1.63, 2.05 and $2.47 \% \mathrm{LIN})$. The productive performance and the egg quality were analyzed according to a completely randomized design in a factorial arranged of $3 \times 3$. The means were compared by Tukey test. Birds fed diets with 0.50 and $0.60 \%$ MET had higher feed intake and egg production $(\mathrm{p}<0.05)$ than those fed diets with $0.40 \%$ MET. There was significant interaction $(\mathrm{p}<0.05)$ between MET and LIN levels in egg weight. A better feed conversion and egg mass was observed for birds fed diets with $0.60 \%$ MET compared to those fed diets with $0.40 \%$ and $0.50 \%$ MET. Egg production and quality $(\mathrm{p}>0.05)$ did not improve with high levels of LIN.
\end{abstract}

Keywords: methionine, linoleic acid, laying quail productivity, egg quality. 


\section{INTRODUCCIÓN}

La formulación de dietas para codornices japonesas se basa en requerimientos nutricionales sugeridos por investigadores a partir de experimentos realizados bajo condiciones climáticas diferentes a las de los andes peruanos. Instituciones dedicadas a la nutrición avícola, desde hace varias décadas, no reportan nuevos informes sobre el requerimiento nutricional de la codorniz (NRC, 1994), hay otras organizaciones de investigación especializada que no reportan ninguna información nutricional para codornices (FEDNA, 2018), sin embargo, a nivel de Sudamérica, Rostagno et al. (2017) han publicado tablas brasileras de requerimientos nutricionales para codornices en crecimiento y fase de postura, lo cual sirve de guía importante para alimentar estas aves, pero cuando se practica la coturnicultura en zonas de clima frío, la ingesta de alimento es diferente en lugares con climas tropicales y en los de clima con temperaturas más bajas, lo cual implica realizar un reajuste de las concentraciones de nutrientes de la dieta, evidenciándose la necesidad de información.

Aunque los requerimientos nutricionales varían considerablemente según la edad, peso del ave y la producción de huevos; Rostagno et al. (2017) sugiere niveles de MET de 0,569 y 0,523\% y niveles de LIN de 0,999 y $1,107 \%$ para codornices en fase de postura con pesos corporales de 190 y $210 \mathrm{~g}$, y con ingestas de alimento de 25,85 y 23,13 g/día, respectivamente. Bello (1997) evaluó los niveles de metionina en dietas para codornices en el período de puesta temprana, encontrando mejor rendimiento con niveles de $0,480 \%$ de MET y 0,692\% de MET + Cisteína (Cis). Del mismo modo se indica que, el LIN en la dieta de la codorniz japonesa se requiere en cantidades de $2 \mathrm{~g} / \mathrm{kg}$ para prevenir problemas de hipertrofia hepática acompañada de acumulación de lípidos (Murai et al., 2007). Otros investigadores encontraron que el aumento de MET + Cis de $0,700 \%$ a $0,875 \%$ redujo el porcentaje de proteína de la yema. Se observó un mayor porcentaje de lípidos en la yema en los huevos de las codornices alimentadas con dietas con $1,050 \%$ de MET + Cis
(García et al., 2005). Los requerimientos de LIN no se terminan de definir, por la variabilidad de resultados encontrados, así Whitehead (1984) recomendaron $1,0 \%$ de LIN en la dieta, mientras que Scragg et al. (1987) encontró ventajas en el peso del huevo con niveles de LIN superiores al $2 \%$.

Los estándares de clasificación del tamaño de huevo de codorniz no existen como sucede para los de gallina ponedora; en el Perú tampoco se da la venta de huevos de codorniz por masa de huevo, sino por número de huevos; por lo que no sería beneficioso económicamente para el productor la obtención de huevo demasiado grande, de la misma manera aparecen mayores problemas en el huevo grande por tener la cáscara débil. Sin embargo, tampoco resulta muy atractivo para el comprador, el huevo demasiado pequeño. Por lo que, el control del tamaño del huevo de codorniz permitirá su manipulación de acuerdo a la conveniencia económica o influenciada por el mercado para lo cual se debe determinar el efecto de los nutrientes dietarios en las características del producto. En ese sentido se ha orientado el presente trabajo, con el objetivo de evaluar tres niveles de metionina, de $0,40 \%$, $0,50 \%$ y $0,60 \%$, y tres niveles de ácido linoleico de $1,63 \%, 2,05 \%$ y $2,47 \%$ en la dieta de codorniz sobre el rendimiento productivo en postura y la calidad del huevo, con énfasis en la incidencia de problemas de calidad de cáscara y en un ambiente no convencional como lo es el de sierra peruana a $2750 \mathrm{msnm}$.

\section{MATERIALES Y MÉTODOS}

\section{Manejo de las aves y dietas experimentales}

El experimento se llevó a cabo en las instalaciones avícolas de la Facultad de Ingeniería en Ciencias Pecuarias (FICP), de la Universidad Nacional de Cajamarca (UNC). Se criaron ochocientas codornices de 1 día de edad, 98\% hembras, procedentes de una granja comercial de Lima-Perú. La fase de crecimiento duró 35 días bajo condiciones similares de manejo y alimentación. A los 42 días de edad, 576 hembras se seleccionaron y trasladaron al galpón de producción y se alojaron en jaulas de $0,60 \times 0,54 \times 0,22 \mathrm{~cm}$ (largo $\mathrm{x}$ 
ancho $\mathrm{x}$ alto), 16 aves por jaula.

Todas las codornices fueron alimentadas con dietas formuladas para tener un contenido similar de energía metabolizable, minerales y aminoácidos, pero diferían en los niveles de MET (0,40 0,50 y 0,60\%), y de LIN (1,63 2,05 y 2,47\%). Se formaron nueve combinaciones de tratamientos con los siguientes contenidos de MET-LIN: $\mathrm{T}_{1}: 0,40-1,63 \%, \mathrm{~T}_{2}: 0,40-2,05 \%, \mathrm{~T}_{3}: 0,40-$ $2,47 \%, \mathrm{~T}_{4}: 0,50-1,63 \%, \mathrm{~T}_{5}: 0,50-2,05 \%, \mathrm{~T}_{6}: 0,50-$
$2,47 \%, \mathrm{~T}_{7}: 0,60-1,63 \%, \mathrm{~T}_{8}: 0,60-2,05 \%, \mathrm{~T}_{9}: 0,60-$ $2,47 \%$. Cada tratamiento con 4 repeticiones, una repetición conformada por 16 aves alojadas en cada jaula. Las dietas se suministraron a voluntad en dos comidas diarias. La composición en ingredientes y el contenido de nutrientes calculados de las dietas se muestran en la Tabla 1. La evaluación duró 24 semanas. Se proporcionó luz durante $16 \mathrm{~h}$ diarias y la temperatura durante el experimento se mantuvo entre $21-23^{\circ} \mathrm{C}$.

Tabla 1. Porcentaje de ingredientes y contenido nutricional calculado de las dietas experimentales, según tratamientos

\begin{tabular}{|c|c|c|c|c|c|c|c|c|c|}
\hline Ingredientes & 1 & 2 & 3 & 4 & 5 & 6 & 7 & 8 & 9 \\
\hline Maíz amarillo & 53,38 & 53,38 & 53,38 & 53,28 & 53,28 & 53,28 & 53,18 & 53,18 & 53,18 \\
\hline Afrecho de trigo & 1,5 & 1,5 & 1,5 & 1,5 & 1,5 & 1,5 & 1,5 & 1,5 & 1,5 \\
\hline Torta de soya $44 \%$ & 33 & 33 & 33 & 33 & 33 & 33 & 33 & 33 & 33 \\
\hline Aceite de palma & 2 & 1 & & 2 & 1 & & 2 & 1 & \\
\hline Aceite de soya & & 1 & 2 & & 1 & 2 & & 1 & 2 \\
\hline Carbonato de calcio & 8,15 & 8,15 & 8,15 & 8,15 & 8,15 & 8,15 & 8,15 & 8,15 & 8,15 \\
\hline Fosfato dicálcico & 1 & 1 & 1 & 1 & 1 & 1 & 1 & 1 & 1 \\
\hline Sal común & 0,4 & 0,4 & 0,4 & 0,4 & 0,4 & 0,4 & 0,4 & 0,4 & 0,4 \\
\hline DLMetionina & 0,07 & 0,07 & 0,07 & 0,17 & 0,17 & 0,17 & 0,27 & 0,27 & 0,27 \\
\hline Lisina $\mathrm{HCl}$ & 0,1 & 0,1 & 0,1 & 0,1 & 0,1 & 0,1 & 0,1 & 0,1 & 0,1 \\
\hline Cloruro de colina $60 \%$ & 0,05 & 0,05 & 0,05 & 0,05 & 0,05 & 0,05 & 0,05 & 0,05 & 0,05 \\
\hline Premix vity $\min { }^{1}$ & 0,1 & 0,1 & 0,1 & 0,1 & 0,1 & 0,1 & 0,1 & 0,1 & 0,1 \\
\hline Zinc bacitracina & 0,05 & 0,05 & 0,05 & 0,05 & 0,05 & 0,05 & 0,05 & 0,05 & 0,05 \\
\hline Absorbente micotox. & 0,1 & 0,1 & 0,1 & 0,1 & 0,1 & 0,1 & 0,1 & 0,1 & 0,1 \\
\hline Antimicótico & 0,1 & 0,1 & 0,1 & 0,1 & 0,1 & 0,1 & 0,1 & 0,1 & 0,1 \\
\hline TOTAL & 100,00 & 100,00 & 100,00 & 100,00 & 100,00 & 100,00 & 100,00 & 100,00 & 100,00 \\
\hline \multicolumn{10}{|l|}{$\begin{array}{l}\text { Contenido nutricional } \\
\text { calculado }\end{array}$} \\
\hline Materia seca, $\%$ & 90,20 & 90,20 & 90,20 & 90,21 & 90,21 & 90,21 & 90,22 & 90,22 & 90,22 \\
\hline Proteína cruda, $\%$ & 20,10 & 20,10 & 20,10 & 20,09 & 20,09 & 20,09 & 20,08 & 20,08 & 20,08 \\
\hline Fibra cruda, $\%$ & 3,78 & 3,78 & 3,78 & 3,78 & 3,78 & 3,78 & 3,77 & 3,77 & 3,77 \\
\hline $\mathrm{EM}, \mathrm{kcal} / \mathrm{kg}$ & 2890 & 2890 & 2890 & 2892 & 2892 & 2892 & 2894 & 2894 & 2894 \\
\hline Lisina, $\%$ & 1,23 & 1,23 & 1,23 & 1,23 & 1,23 & 1,23 & 1,23 & 1,23 & 1,23 \\
\hline Metionina, $\%$ & $\mathbf{0 , 4 0}$ & $\mathbf{0 , 4 0}$ & $\mathbf{0 , 4 0}$ & $\mathbf{0 , 5 0}$ & $\mathbf{0 , 5 0}$ & $\mathbf{0 , 5 0}$ & 0,60 & 0,60 & 0,60 \\
\hline Triptófano, \% & 0,27 & 0,27 & 0,27 & 0,27 & 0,27 & 0,27 & 0,27 & 0,27 & 0,27 \\
\hline Pdisponible, $\%$ & 0,35 & 0,35 & 0,35 & 0,35 & 0,35 & 0,35 & 0,35 & 0,35 & 0,35 \\
\hline $\mathrm{Ca}, \%$ & 3,38 & 3,38 & 3,38 & 3,38 & 3,38 & 3,38 & 3,38 & 3,38 & 3,38 \\
\hline Ácidolinoleico, \% & 1,63 & 2,05 & 2,48 & 1,63 & 2,05 & 2,47 & 1,63 & 2,05 & 2,47 \\
\hline
\end{tabular}

Niveles por kg del Premix de vitaminas y minerales: Vitamina A 10000,000 UI; Vitamina D3 3000000 UI; Vitamina E 15000 UI; Vitamina K3 2,5 g; Riboflavina 4 g; Cianocobalamina 12 mg; Ácido pantoténico 6 g; Ácido fólico 500 mg; Niacina 20 g. Manganeso 60 g; Zinc 45 g; Hierro 40 g; Cobre $5 \mathrm{~g}$; Iodo $1 \mathrm{~g}$; Selenio $100 \mathrm{mg}$.

\section{Rendimiento productivo y calidad del huevo}

La producción de huevos, el número de huevos rotos y sin cáscara, la ingesta de alimento y la mortalidad se registraron diariamente por repetición. Se determinó producción de huevo $\left(\%\right.$ postura $=\mathrm{N}^{\circ}$ de huevos pro- ducidos $/ \mathrm{N}^{\circ}$ de aves vivas x 100), ingesta de alimento, peso medio de huevo, masa de huevo (peso medio de huevo $\mathrm{x} \%$ de postura), conversión alimenticia (ingesta de alimento/masa de huevo), huevos con cáscara defectuosa $\left(\mathrm{N}^{\circ}\right.$ de huevos rotos y sin cáscara $/ \mathrm{N}^{\circ}$ de 
huevos total x 100), peso relativo de la cáscara del huevo. Estos indicadores se calcularon por período de 28 días y globalmente. El peso de los huevos se realizó en una balanza marca KERN de $400 \mathrm{~g}$ de capacidad y 0,01g de precisión

\section{Análisis estadístico}

El rendimiento productivo y el número de huevos defectuosos se analizó según un diseño completamente randomizado en arreglo factorial de 3 x 3 (tres niveles de metionina y tres niveles de ácido linoleico) con 4 repeticiones y 16 aves por repetición. El peso de la cáscara determinó con cinco huevos recogidos por repetición durante tres días al final de cada período de 28 días. Todos los datos fueron analizados por ANOVA (SAS Institute, 1998) y la prueba de Tukey (p $<0,05)$ se utilizó para comparar las medias significativamente diferentes.

\section{RESULTADOS Y DISCUSIÓN}

\section{Rendimiento productivo}

Los resultados de rendimiento de las codornices japonesas sometidas a los tratamientos experimentales se muestran en la Tabla 2. Los niveles de metionina ( $p$ $<0,05)$ afectaron significativamente el porcentaje de puesta. No hubo efectos significativos de los niveles de ácido linoleico y no hubo interacción significativa entre los factores, excepto en el indicador peso del huevo. Las aves alimentadas con un $0,40 \%$ de MET en la dieta tuvieron una producción disminuida de huevos en comparación con las otras aves. A pesar de que las aves alimentadas con $0,60 \%$ de MET mostraron tendencia a una mayor producción de huevos que las aves alimentadas con $0,50 \%$ de MET, no hubo diferencias. Estos resultados coinciden con algunos estudios previos que han informado de una producción significativamente mayor de huevos con niveles más altos de MET (Schafer et al., 1996; Bello, 1997). La producción de huevo no se vio afectada por los niveles de LIN en la dieta, aunque la producción tendió a ser mayor con el aumento de LIN.

El aumento en el nivel de MET de 0,40\% a 0,50\% propició un mayor consumo de alimento, lo cual no se observó cuando se incrementó los niveles dietarios de

Tabla 2. Efectos de los niveles de metionina y ácido linoleico en el rendimiento de las codornices japonesas

\begin{tabular}{cccccc}
\hline $\begin{array}{c}\text { Niveles de } \\
\text { Nutrientes }\end{array}$ & $\begin{array}{c}\text { Producción } \\
\text { dehuevos(\%) }\end{array}$ & $\begin{array}{c}\text { Ingesta de } \\
\text { alimento(g) }\end{array}$ & $\begin{array}{c}\text { Peso del } \\
\text { huevo(g) } \mathbf{1}^{\mathbf{1}}\end{array}$ & $\begin{array}{c}\text { Masa de } \\
\text { huevo(g) }\end{array}$ & $\begin{array}{c}\text { Conversión } \\
\text { alimenticia }\end{array}$ \\
\hline Metionina (\%) & & & & & \\
\hline 0,40 & $74,19^{\mathrm{A}}$ & $26,45^{\mathrm{A}}$ & 11,31 & $8,38^{\mathrm{A}}$ & $3,15^{\mathrm{B}}$ \\
0,50 & $75,84^{\mathrm{B}}$ & $27,08^{\mathrm{B}}$ & 11,54 & $8,72^{\mathrm{B}}$ & $3,10^{\mathrm{AB}}$ \\
0,60 & $77,64^{\mathrm{B}}$ & $27,29^{\mathrm{B}}$ & 11,70 & $9,05^{\mathrm{C}}$ & $3,01^{\mathrm{A}}$ \\
Ácido linoleico(\%) & & & & & 3,11 \\
1,63 & 75,35 & 26,87 & 11,46 & 8,74 & 3,08 \\
2,05 & 75,62 & 26,92 & 11,58 & 8,68 & 3,10 \\
\hline
\end{tabular}

Medias seguidas por letras diferentes en la columna son diferentes según la prueba de Tukey $(\mathrm{p}<0,05)$.

Interacción significativa Metionina: Ácido linoleico.

$0,50 \%$ a $0,60 \%$ de MET, lo que podría coincidir con lo reportado por Raes et al. (2002) quien indica que los niveles de MET dietaria no influyen en el aumento del consumo de alimento, advirtiéndose que, cuando se analiza el consumo de alimento de una especie animal es necesario considerar los niveles de otros nutrientes en las dietas, como el de los nutrientes que aportan energía, y si se trata de aves ponedoras, los niveles de calcio; así Murakami et al. (1994) fue tajante al concluir que los niveles de MET en la dieta no tienen ningún efecto sobre el alimento consumido por codornices en el período de puesta. En lo que concierne al otro nutriente evaluado, el consumo de alimento no se vio afectado por el aumento en los niveles de LIN, lo que coincide con las conclusiones de Vilchez et al. (1991) quien evaluó niveles altos de LIN y de otros ácidos 
grasos dietarios, concluyendo que el LIN no es influyente en el consumo de alimento de las codornices a diferencia de otros ácidos como el palmítico.

Hubo una interacción significativa entre la MET y los niveles de LIN en la dieta (Tabla 3) sobre el peso del huevo, lo que indica que los principales factores son dependientes. Las aves alimentadas con 0,60\% de MET y $2,05 \%$ de LIN tuvieron un mayor peso del huevo que las aves alimentadas con $1.63 \%$ de LIN, mientras que las aves alimentadas con $2,47 \%$ mostra- ron valores intermedios. Dentro de 0,40 y $0,60 \%$ de MET, el peso del huevo no se vio afectado por los niveles de LIN. Con $1,63 \%$ de LIN, los huevos eran más pesados cuando las aves fueron alimentadas con 0,60\% de MET. Dentro del nivel LIN de 2,05\%, los huevos fueron más pesados a 0,50 y $0,60 \%$ de MET. Finalmente, el aumento en los niveles de MET de las dietas que contenían $2,47 \%$ de LIN aumentó el peso del huevo.

Tabla 3. Efectos simples de la interacción Metionina: Ácido linoleico en el peso del huevo de la codorniz ponedora

\begin{tabular}{ccccc}
\hline \multirow{2}{*}{ Acido linoleico (\%) } & \multicolumn{3}{c}{ Metionina(\%) } & Media \\
\cline { 2 - 5 } & 0,4 & 0,5 & $11,71^{\mathrm{Ab}}$ & 11,46 \\
\hline 1,63 & $11,27^{\mathrm{Aa}}$ & $11,40^{\mathrm{Aa}}$ & $11,76^{\mathrm{Ab}}$ & 11,58 \\
2,05 & $11,33^{\mathrm{Aa}}$ & $11,65^{\mathrm{Bb}}$ & $11,62^{\mathrm{Ab}}$ & 11,51 \\
2,47 & $11,34^{\mathrm{Aa}}$ & $11,56^{\mathrm{ABab}}$ & 11,70 \\
\hline Media & 11,31 & 11,54 & \\
\hline
\end{tabular}

Medias seguidas por letras mayúsculas diferentes en la columna son diferentes por la prueba de Tukey $(\mathrm{p}<0,05)$

Medias seguidas por letras pequeñas diferentes en la fila son diferentes por la prueba de Tukey $(\mathrm{p}<0,05)$

Los niveles de MET más altos en la dieta mejoraron la masa de huevos. Los efectos de los niveles de MET en la masa de huevo pueden reflejar la mayor tasa de postura y el peso del huevo que también se observaron con el aumento de los niveles de MET, ya que la masa de huevo está directamente relacionada con estos dos parámetros. Cabe señalar que los niveles de MET más altos que estos podrían dar como resultado una mayor masa de huevos. De hecho, la maximización de la masa de huevos producida se ha observado en algunos estudios con 0,60\% de MET (Pinto, 1998). Por otro lado, Bello (1997) reportó un aumento en la producción de huevos y el peso del huevo cuando se usaron niveles de aminoácidos azufrados de hasta 0,692 y $0,839 \%$ en la dieta. No hubo efectos significativos de los niveles de LIN en la masa de huevos.

No hubo un efecto significativo de la MET y LIN, o de la interacción entre estos factores, en la conversión alimenticia. La conversión alimenticia ( $\mathrm{kg}$ de alimento: $\mathrm{kg}$ de huevos) se mejoró con un $0,60 \%$ de Met y se obtuvieron peores resultados con un $0,40 \%$. La conversión alimenticia con $0,50 \%$ de Met no fue estadísticamente diferente de los otros tratamientos. Al respecto, investigadores atribuyen mejoras en la conversión alimenticia por efectos de los niveles de proteína en la dieta (Pinto, 1998), pudiéndose mejorar la conversión alimenticia por el aumento en los niveles de proteína, pero considerando los niveles de aminoácidos azufrados, encontrando este investigador una mejora en la conversión alimenticia por masa de huevo con hasta $22 \%$ de PC en la dieta. Similares resultados fueron corroborados parcialmente por Bello (1997) quien también mejoró la conversión alimenticia en función de MET + Cys. Murakami (1994) observó una mejor conversión alimenticia por masa de huevo con niveles de proteína de dieta entre 19 y $20 \%$, lo que sirvió como referencia en el presente trabajo para considerar las dietas isoproteicas en base a $20 \%$ de proteína cruda. Por otro lado, los niveles de LIN no produjeron diferencias en los datos de conversión alimenticia.

\section{Calidad de huevo}

Los efectos de los tratamientos sobre la calidad del huevo se muestran en la Tabla 4. No hubo efectos significativos del tratamiento sobre el porcentaje de cáscara de huevo. Murakami et al. (1994) también encontraron que los niveles de MET no tenían ningún efecto sobre este parámetro. Por otro lado, Bello (1997) informó de la disminución del porcentaje de 
cáscara de huevo con niveles crecientes de MET en la dieta, pero cuando el huevo incrementó de peso. Kim et al. (2007) encontró mayor espesor de cáscara en huevos de aves ponedoras alimentadas con $2 \%$ de LIN, que con dietas con bajos niveles de LIN.

\section{CONCLUSIONES}

La producción de huevo, ingesta de alimento y masa de huevo, se incrementaron con dietas con $0.50 \%$ de metioni- na en comparación con dietas que contenían $0.40 \%$ de metionina, aunque no hubo efectos sobre la conversión alimenticia.

El rendimiento de la producción o la calidad del huevo no mejoraron con niveles de ácido linoleico de 2,05 y $2,47 \%$.

Tabla 4. Efectos de los niveles de metionina y ácido linoleico en la calidad del huevo de la codorniz ponedora

\begin{tabular}{|c|c|c|}
\hline Niveles de Nutrientes & Peso de cáscara * $(\%)$ & Huevos defectuosos (\%) \\
\hline \multicolumn{3}{|l|}{ Metionina(\%) } \\
\hline 0,40 & $8,56^{\mathrm{b}}$ & 2,43 \\
\hline 0,50 & $8,63^{\mathrm{ab}}$ & 2,45 \\
\hline 0,60 & $8,67^{\mathrm{ab}}$ & 2,47 \\
\hline \multicolumn{3}{|l|}{ Ácido linoleico (\%) } \\
\hline 1,63 & 8,63 & 2,39 \\
\hline 2,05 & 8,62 & 2,43 \\
\hline 2,47 & 8,60 & 2,41 \\
\hline
\end{tabular}

Medias seguidas por letras diferentes en la columna son diferentes según la prueba de Tukey $(\mathrm{p}<0,05)$,

*Peso de cascara $(\%)=$ peso de la cascara $(\mathrm{g}) /$ peso del huevo $(\mathrm{g}) \mathrm{X} 100$

\section{REFERENCIAS BIBLIOGRÁFICAS}

Bello, M. 1997. "Níveis de energia metabolizável e de metionina em rações de codornas japonesas (Coturnix coturnix japonica) na fase inicial de postura" En: Escola Superior de Agricultura de Lavras; Minas Gerais (Brasil).

FEDNA. 2018. Necesidades nutricionales para avicultura. Madrid (España): Fundación Española para el Desarrollo de la Nutrición Animal.

Garcia E. A., A. A. Mendes, C .C Pizzolante, E. S. Saldanha, J. Moreira, C. Mori, y A. C. Pavan. 2005. "Protein, Methionine+Cystine and Lysine Levels for Japanese Quails During the Production Phase." Brazilian Journal of Poultry Science 7 (1): 11-18

Harms R.H. y G.B. Russell. 2004. "Performance of commercial laying hens when fed diets with various sources of energy." Journal Applied Poultry Research 13:365-369

Kim J. H., J. Hwangbo, N. J. Choi, H. G. Park, D. H. Yoon, E. W. Park, S. H. Lee, B. K. Park, y Y. J. Kim. 2007. "Effect of Dietary Supple- mentation with Conjugated Linoleic Acid, with Oleic, Linoleic, or Linolenic Acid, on Egg Quality Characteristics and Fat Accumulation in the Egg Yolk." Journal Poultry Science 86: 1180-1186

Liu Y., X. Lin, X. Zhou, D. Wan, Z. Wang, X. Wu, y Y. Yin. 2017. "Effects of dynamic feeding low and high methionine diets on egg quality traits in laying hens.” Journal Poultry Science 96: 1459-1465

Murai A, M. Furuse, y J. Okumura. 2007. "Role of dietary $\gamma$-linolenic acid in liver lipid metabolism in Japanese quail." Journal British Poultry Science 36 (5): 821-827

Murakami, A., A. Furlan, A. Tateishi, K. Kira, y R. Ribeiro. 1994. "Níveis de metionina para codornas japonesas (Coturnix coturnix japonica) em postura." En 31 Reunião Anual da Sociedade Brasileira de Zootecnia. Maringá (Brasil).

NRC (National Research Council). 1994. Nutrient requirements of poultry. Washington, D.C (USA): National Academy of Science. 
Pinto, R. 1998. Níveis de proteína e energia para codornas japonesas (Coturnix coturnix japonica) em postura Tesis de Grado. Universidade Federal de Viçosa. Viçosa (Brasil)

Safaa H. M., M. P. Serrano, D. G. Valencia, X. Arbe, E. Jiménez-Moreno, R. Lázaro, y G. G. Mateos. 2008. "Effects of the Levels of Methionine, Linoleic Acid, and Added Fat in the Diet on Productive Performance and Egg Quality of Brown Laying Hens in the Late Phase of Production." Poultry Science 87: 1595-1602

SAS Institute. 1998. SAS STAT User's Guide: Version 6thed. SAS Inst. Inc., Cary, NC.

Scragg R. H., N. B. Logan, y N. Geddes. 1987. "Response of egg weight to the inclusion of various fats in layer diets." Journal British Poultry Science. 28: 15-21

Shafer D. J, J. B. Carey, y J. F. Prochaska. 1996. "Effect of Dietary Methionine Intake on Egg Component Yield and Composition." Journal Poultry Science 75: 1080-1085

Sohail S. S., M. M. Bryant, y D. A. Roland. 2002. "Influence of supplemental lysine, isoleucine, threonine, tryptophan, and total sulphur amino acids on egg weight of Hy-Line W36 hens." Journal Poultry Science. 81:1038-1044

Raes K., G. Huyghebaert, S. De Smet, L. Nollet, S. Arnouts, y Demeyer. 2002. "The deposition of conjugated linoleic acids in eggs of laying hens fed diets varying in fat level and fatty acid profile." Journal Animal Nutrition 132 (2): 182-189

Rostagno, H., L. Teixeira, M. Hannas, J. Lopes, N. Sakomura, F. Perazzo, A. Saraiva, M. Teixeira de Abreu, P. Borges, R. de Oliveira, S. de Toledo, y C. de Oliveira. 2017. Tablas Brasileñas para Aves y Cerdos. Composición de Alimentos y Requerimientos Nutricionales. Viçosa (Brasil): Universidad Federal de Viçosa. Departamento de Zootecnia.

Vilchez C., S. P. Touchburn, E. R. Chavez, y C. W.
Chan. 1991. "Effect of Feeding Palmitic, Oleic, and Linoleic Acids to Japanese Quail Hens (Coturnix coturnix japonica). 1. Reproductive Performance and Tissue Fatty Acids." Canadian Journal Poultry Science 70: 24842493

Whitehead C. C. 1984. "Essential fatty acids in poultry nutrition". En Fats in Animal Nutrition. J. Wiseman, (ed). Londres (Inglaterra): Butterworths. 